\title{
EXPLICIT BOUNDS FOR HERMITE POLYNOMIALS IN THE OSCILLATORY REGION
}

\author{
WILLIAM H. FOSTER AND ILIA KRASIKOV
}

\begin{abstract}
We apply a method of positive quadratic forms based on polynomial inequalities to establish sharp explicit bounds on the envelope of Hermite polynomials in the oscillatory region $|x|<(2 k-3 / 2)^{1 / 2}$.
\end{abstract}

\section{Introduction}

In this paper we use a standard normalization of the Hermite polynomials; that is, they are defined by $H_{1}=0, H_{0}=1$ and the recurrence $H_{k+1}=2 x H_{k}+2 k H_{k-1}$.

It has been known for a long time (see [11, Section 8.22]; also [1]) that the asymptotics for Hermite polynomials $H_{k}(x)$ imply that in the oscillatory region

$$
e^{-x^{2}} \sqrt{1-x^{2} /(2 k+1)}\left(H_{k}(x)\right)^{2}
$$

behaves much like a cosine of an appropriate real function. Thus its envelope is almost independent of $x$. This result was generalized to a large family of continuous weights, and an excellent overview of recent results can be found in $[2,3,4,8,9,10]$. It has been observed in $[6,7]$ that instead of asymptotics or potential theory one may, whenever we have a second-order differential equation or its difference analogue, use simple polynomial inequalities; for example,

$$
\left(p^{\prime}(x)\right)^{2}-p(x) p^{\prime \prime}(x) \geqslant 0,
$$

which is valid for all real root polynomials. This approach easily yields explicit bounds rather than asymptotics for extreme roots, and an envelope of orthogonal polynomials in the oscillatory region. For instance, this method works for the Bessel function and the Krawtchouck polynomials [6], in addition to the classical polynomials. Since in the case of Krawtchouck polynomials the weight is discontinuous, this is certainly not covered by the general theory. Moreover, it turns out that by slightly perturbing the basic inequality, one can obtain very sharp bounds for extreme roots [5]. The aim of this paper is to illustrate the method for the case of the Hermite polynomials by finding a tight bound for its envelope in the oscillatory region. Our principal result is as follows.

Theorem 1. For $x^{2}<2 k-3 / 2$,

$$
\left(H_{k}(x)\right)^{2} e^{-x^{2}} \leqslant C(k) \frac{2 y^{2}-4 y+3}{\sqrt{y\left(4 y^{4}-12 y^{3}+9 y^{2}+10 k y-12 k\right)}} \exp \left(\frac{15 x^{2}}{2 y(2 y-3)^{2}}\right)
$$

where $y=2 k-x^{2}$,

$$
C(k)=\frac{2 k \sqrt{4 k-2} k !^{2}}{\sqrt{8 k^{2}-8 k+3}(k / 2) !^{2}} \quad \text { for } k \text { even, }
$$


Explicit bounds for Hermite polynomials in the oscillatory region

and

$$
C(k)=\frac{\sqrt{16 k^{2}-16 k+6} k !(k-1) !}{\sqrt{2 k-1}((k-1) / 2) !^{2}} \quad \text { for } k \text { odd } .
$$

The theorem is 'sharp' in the sense that if

$$
\exp \left(\frac{15 x^{2}}{2 y(2 y-3)^{2}}\right) \text { is replaced by } \exp \left(-\frac{15 x^{2}}{2 y(2 y-3)^{2}}\right)
$$

the inequality reverses at all roots of the equation

$$
x y(2 y-3) H_{k}(x)=\left(2 y^{2}-4 y+3\right) H_{k-1}(x) .
$$

Thus, for $x$ not too close to $(2 k)^{1 / 2}$ the theorem gives an envelope of $\left|H_{k}(x)\right|$ with accuracy $O\left(1 / k^{2}\right)$. Figure 1 shows the graphs of $\left(H_{24}(x)\right)^{2} e^{-x^{2}}$ and the envelope given by the above theorem for $k=24$.

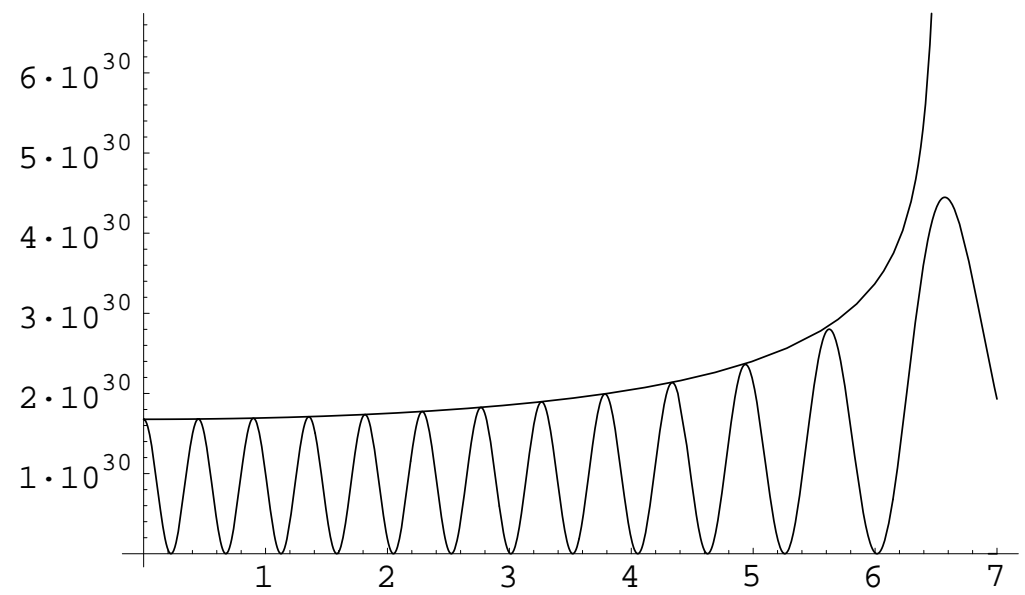

Figure 1: Envelope of $\left(H_{24}(x)\right)^{2} e^{-x^{2}}$.

\section{Proofs}

We start with a generalization of inequality (1).

Lemma 1. Let $p=p(x)$ be a real polynomial having only real roots. Then, for any integral $m \geqslant 0$,

$$
U_{2 m}(p)=\frac{1}{2} \sum_{j=0}^{2 m}(-1)^{m+j}\left(\begin{array}{c}
2 m \\
j
\end{array}\right) p^{(j)} p^{(2 m-j)} \geqslant 0,
$$

where $p^{(i)}=d^{i} p / d x^{i}$.

Proof. Note that for $m=1$ this is just inequality (1). We present two proofs for $m \neq 1$. 
First proof. The result follows by double induction on $m$ and the degree of the polynomial from the easily checked identity

$$
U_{2 m}\left(\left(x-x_{0}\right) p\right)=\left(x-x_{0}\right)^{2} U_{2 m}(p)+2 m(2 m-1) U_{2 m-2}(p) .
$$

Second proof. Let $s_{t}\left(x_{1}, \ldots, x_{n}\right)=\sum_{\left\{i_{1}, \ldots, i_{t}\right\} \subset\{1, \ldots, n\}} x_{i_{1}} \ldots x_{i_{t}}$ be the symmetric function of homogeneous degree $t$. We observe that if $p(x)=\left(x-a_{1}\right) \ldots\left(x-a_{n}\right)$ then

$$
p^{(t)}=t ! s_{n-t}\left(x-a_{1}, \ldots, x-a_{n}\right) .
$$

On using the identity

$\sum_{j=0}^{2 m}(-1)^{m+j} s_{j}\left(x-a_{1}, \ldots, x-a_{n}\right) s_{2 m-j}\left(x-a_{1}, \ldots, x-a_{n}\right)=s_{m}\left(\left(x-a_{1}\right)^{2}, \ldots,\left(x-a_{n}\right)^{2}\right)$,

we obtain

$$
U_{2 m}(p)=\frac{(2 m) ! s_{m}\left(\left(x-a_{1}\right)^{2}, \ldots,\left(x-a_{n}\right)^{2}\right)}{2} \geqslant 0 .
$$

In the sequel we deal with the case where $m=2$, so we set

$$
U(p)=U_{4}(p)=3\left(p^{\prime \prime}\right)^{2}-4 p^{\prime} p^{\prime \prime \prime}+p p^{(4)} \geqslant 0 .
$$

To simplify the notation we put $y=2 k-x^{2}, u=H_{k}(x)$ and $v=H_{k}^{\prime}(x)$. We assume that $|x|<(2 k)^{1 / 2}$, and consider the following form:

$$
V(x)=U(u)+\frac{6 v^{2}}{2 k-x^{2}} \geqslant 0 .
$$

Lemma 2. For $k$ even,

$$
V(0)=\frac{8 k(2 k-1) k !^{2}}{(k / 2) !^{2}}
$$

For $k$ odd,

$$
V(0)=\frac{4\left(8 k^{2}-8 k+3\right) k !(k-1) !}{((k-1) / 2) !^{2}} .
$$

Proof. This follows by a direct calculation from $H_{k}(0)=(-1)^{m}(2 m) ! / m$ ! for $k=2 m$ even, $H_{k}(0)=0$ for $k$ odd, and $H_{k}^{\prime}(x)=2 k H_{k-1}(x)$.

The following lemma is the core of our method.

Lemma 3. For $x^{2} \geqslant 2 k-3 / 2$,

$$
\left|\ln \frac{V(x)}{V(0)}-x^{2}-\frac{1}{2} \ln \frac{4 y^{4}-12 y^{3}+9 y^{2}+10 k y-12 k}{2 y(2 k-1)\left(8 k^{2}-8 k+3\right)}\right| \leqslant \frac{15 x^{2}}{2 y(2 y-3)^{2}} .
$$

Proof. Using the differential equation $H_{k}^{\prime \prime}=2 x v-2 k u$, we may express higher derivatives of $H_{k}(x)$ in $v$ and $u$, as follows:

$$
\begin{aligned}
H_{k}^{\prime \prime \prime}(x) & =\left(2-2 k+4 x^{2}\right) v-4 k u, \\
H_{k}^{(4)} & =4 x(3-2 k+2 x) v+2 k\left(2 k-4-4 x^{2}\right) u .
\end{aligned}
$$


Then $V(x)$ and $V^{\prime}(x)=d V / d x$ become quadratic forms in $v$ and $u$ :

$$
\begin{aligned}
V(x) & =8 k(y-1) u^{2}-4 x(2 y-3) v u+\frac{2\left(2 y^{2}-4 y+3\right)}{y} v^{2}, \\
V^{\prime}(x) & =8 x k(2 y-5) u^{2}-\frac{4 x^{2}\left(4 y^{2}-12 y+3\right)}{y} v u+\frac{4 x\left(2 y^{3}-7 y^{2}+6 y+3\right)}{y^{2}} v^{2} .
\end{aligned}
$$

Now consider the following expression $W=V^{\prime}-z V$, and choose $z=z(x)$ in such a way that the discriminant of $W$ as a quadratic form in $v$ and $u$ vanishes. This yields

$$
z_{1,2}=2 x-\frac{3 x\left(4 k+3 y^{2}-8 y^{3}+4 y^{4}\right) \pm 3 x \sqrt{2 k \Delta(y)}}{y g(y)}
$$

where

$$
\begin{aligned}
\Delta(y) & =8 k-60 k y+18 y^{2}+50 k y^{2}-16 y^{3}, \\
g(y) & =4 y^{4}-12 y^{3}+9 y^{2}+10 k y-12 k .
\end{aligned}
$$

We claim that $W \leqslant 0$ for $z=z_{1}$ and $W \geqslant 0$ for $z=z_{2}$. By $V(x) \geqslant 0$ this is equivalent to

$$
z_{2}<\frac{V^{\prime}}{V}<z_{1}
$$

provided that $x^{2} \leqslant 2 k-3 / 2$. Indeed, for $z$ to be real we need $\Delta(y) \geqslant 0$.

Calculations give $\Delta((3 \pm \sqrt{5}) / 5)>0, \Delta(3 / 5)<0, \Delta(2 k)>0$, and $\Delta(25 k / 8)<0$. Hence $\Delta(y)$ is positive for $(3+\sqrt{5}) / 5 \leqslant y \leqslant 2 k$. Since $(3+\sqrt{5}) / 5<3 / 2$, this means that $z$ is real for $x^{2} \leqslant 2 k-3 / 2$. Furthermore, with the above choice of $z$ the sign of $W$ is determined by the sign of the coefficient at $u^{2}$, which is

$$
\frac{24 x k\left(2 y^{3}-3 y^{2}-10 k y^{2}+16 k y-4 k \pm \sqrt{2 k}(1-y) \sqrt{\Delta(y)}\right)}{y g(y)} .
$$

Notice that for $y \geqslant 3 / 2$, the denominator of this expression is positive, whereas the numerator is negative for $z=z_{1}$ and positive for $z=z_{2}$. This is because

$$
2 k(1-y)^{2} \Delta(y)-\left(2 y^{3}-3 y^{2}-10 k y^{2}+16 k y-4 k\right)^{2}=y x^{2} g(y),
$$

and $g(y+3 / 2)$ is a polynomial with positive coefficients.

Now, by integrating inequality (5) we get

$$
\left|\ln \frac{V(x)}{V(0)}-x^{2}-\frac{1}{2} \ln \frac{g(y)}{2 y(2 k-1)\left(8 k^{2}-8 k+3\right)}\right| \leqslant 3 \sqrt{2 k} \int_{0}^{x} \frac{x \sqrt{\Delta(y)}}{y g(y)} d x .
$$

Denote the last integral by $R$. To estimate $R$, observe that $g(y) \geqslant y^{2}(2 y-3)^{2}$, and $\Delta \leqslant 50 k y^{2}$, for $y \geqslant 3 / 2$. Hence

$$
R \leqslant 30 k \int_{0}^{x} \frac{x d x}{y^{2}(2 y-3)^{2}}=\frac{5 x^{2}(16 k y-6 y-12 k-9)}{6 y(4 k-3)(2 y-3)}-\frac{20 k}{9} \ln \left(1+\frac{3 x^{2}}{2 k(2 y-3)}\right) .
$$

Using $\ln (1+\epsilon) \geqslant \epsilon-\epsilon^{2} / 2$ we get:

$$
R \leqslant \frac{15 x^{2}\left(4 k^{2}-2 k y+y^{2}-3 k\right)}{2 k y(4 k-3)(2 y-3)^{2}} \leqslant \frac{15 x^{2}}{2 y(2 y-3)^{2}} .
$$


Proof of Theorem 1. Rewriting equation (4) in the form

$$
V(x)=\frac{2\left(x y(2 y-3) u-\left(2 y^{2}-4 y+3\right) v\right)^{2}}{2 y\left(2 y^{2}-4 y+3\right)}+\frac{2 g(y)}{2 y^{2}-4 y+3} u^{2},
$$

we obtain

$$
u^{2} \leqslant \frac{2 y^{2}-4 y+3}{2 g(y)} V(x),
$$

and the result readily follows from Lemmas 2 and 3.

\section{Final remarks}

Remark 1. Using the original form $U$ instead of $V$ leads to an error term of order $O(1 / k)$ for $H_{k}(x)$. To find the correction $6 v^{2} /\left(2 k-x^{2}\right)$ we added to $U$ a general nonnegative quadratic form in $v$ and $u$ with coefficients depending on $x$. It turns out that the leading coefficient of $W$ weakly depends on the choice of the unknown coefficients. Therefore, to make the difference $\left|z_{1}-z_{2}\right|$ as small as possible, we minimized the order of the discriminant of $W$ in $k$. Surprisingly, this gives instead of the expected differential equation, an algebraic one.

Remark 2. One can use $U$ for obtaining inequalities on $H_{k}(x)$ outside the oscillatory region; observe that whenever the discriminant $\Delta=2 k-y(2 y-3)^{2}$, of $U$ as a quadratic in $v$ and $u$ is positive, $v / u$ must lie within certain bounds. It also yields a good upper bound on the largest zero $x_{1}$ of $H_{k}(x)$, as inside the oscillatory region $v / u$ is unbounded; therefore we should have there $\Delta \leqslant 0$. The solution $x_{0}$ of this equation indeed gives an upper bound

$$
x_{1}<x_{0}=\frac{(s-1) \sqrt{s+1}}{\sqrt{2} s^{1 / 3}}<\sqrt{2 k}-2^{-11 / 6} k^{-1 / 6},
$$

where $s=(\sqrt{k}+\sqrt{k-1})^{4 / 3}$. Yet some simple calculations are needed to justify this because the coefficients of $U$ depend on $x$; see $[5,6]$. In this way we get

$$
\begin{array}{ll}
\frac{H_{k}^{\prime}(x)}{H_{k}(x)}<\frac{4 k(y-1)}{x(2 y-3)+\sqrt{\Delta}}, & x>x_{0} \\
\frac{H_{k}^{\prime}(x)}{H_{k}(x)}>\frac{x(2 y-3)+\sqrt{\Delta}}{2 y-4}, & x>\sqrt{2 k-2 .}
\end{array}
$$

The first inequality is tight only on a small interval around $x=(2 k)^{1 / 2}$, whereas (as one can check) the second is sharp for $x>(2 k)^{1 / 2}$; see Figure 2 for $k=24$.

Remark 3. There is no analogue of Lemma 2 (except the ultraspherical case) for other families of orthogonal polynomials. However, notice that for the classical polynomials one can avoid using orthogonality relations. For example, in our case $y V(x)$ can be expressed by the three-term recurrence relation and $v=2 k H_{k-1}(x)$, as $\sum_{j=k-3}^{k+3} a_{i j} H_{i}(x) H_{j}(x)$, with $a_{i j}$ depending only on $k$. Then one can estimate an initial value by

$$
\int_{-\infty}^{\infty} y V(x) e^{-x^{2}} d x=\sum_{i=-3}^{3} a_{i i}\left\|H_{i}(x)\right\|^{2},
$$

together with bounds on $V(x) / V(0)$. We omit the details. 


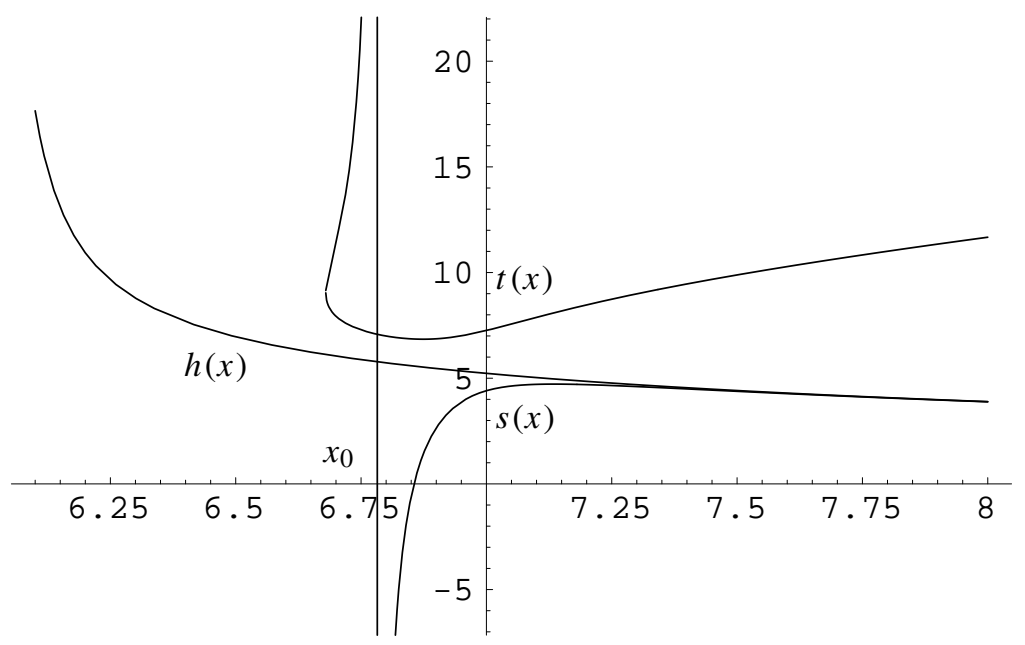

Figure 2: Bounds on $h(x)$.

$$
h(x)=\frac{H_{k}^{\prime}(x)}{H_{k}(x)}, \quad t(x)=\frac{4 k(y-1)}{x(2 y-3)+\sqrt{\Delta}}, \quad s(x)=\frac{x(2 y-3)+\sqrt{\Delta}}{2 y-4}
$$

Remark 4. Although we did not use it in this paper, it worth noticing that an upper bound on $U(p)$ readily yields an upper bound on $p^{2}$, as follows.

Corollary 1. Let $p=a \prod_{j=1}^{k}\left(x-x_{j}\right)$ be a real polynomial with only real roots; then

$$
U(p) \geqslant 6 k(k-1) a^{4 / k} p^{2-4 / k} .
$$

Proof. Let $\mu_{i}=\sum_{j=1}^{k}\left(x-x_{j}\right)^{-i}$; then using $p^{\prime}=p \mu_{1}$ we get

$$
U(p)=6 p^{2}\left(\mu_{2}^{2}-\mu_{4}\right)=12 p^{2} \sum_{j=1}^{k} \sum_{l=1}^{j-1}\left(\left(x-x_{j}\right)\left(x-x_{l}\right)\right)^{-2} .
$$

By the arithmetic-geometric mean this does not exceed

$$
6 k(k-1)\left(\prod_{j=1}^{k}\left(x-x_{j}\right)^{-2 k+2}\right)^{1 /\left(\begin{array}{l}
k \\
2
\end{array}\right)}=6 k(k-1) a^{4 / k} p^{2-4 / k}
$$

Notice also that for a polynomial of a given degree $k$ the inequality (1) can be slightly strengthened as

$$
(k-1) p^{\prime 2}-k p p^{\prime \prime}=\left(k \mu_{2}-\mu_{1}^{2}\right) p^{2} \geqslant 0
$$


In this connection we should like to make the following two conjectures, the first of which generalizes this inequality and sharpens inequality (2) as follows.

Conjecture 1. Let $p=p(x)$ be a real polynomial of degree $k$ having only real roots. Then for any integral $m$, where $1 \leqslant m \leqslant k$,

$$
\frac{1}{2}\left(\begin{array}{c}
k-m \\
m
\end{array}\right)\left(p^{(m)}\right)^{2}+\sum_{j=0}^{m-1}(-1)^{m+j} \frac{\left(\begin{array}{c}
k-j \\
2 m-j
\end{array}\right)}{\left(\begin{array}{c}
k-m \\
m-j
\end{array}\right)}\left(\begin{array}{c}
m \\
j
\end{array}\right) p^{(j)} p^{(2 m-j)} \geqslant 0 .
$$

We call a form $T(p)=\sum_{j=0}^{m} a_{j} p^{(j)} p^{(m-j)}$, where $a_{j}$ may depend only on $m$ and $k=\operatorname{deg}(p)$, a plus form if it is nonnegative for all polynomials $p$ with only real roots.

The plus form $T(p)$ is minimal if for any nonzero plus form $S(p)$ not proportional to $T(p)$, the form $T(p)-S(p)$ is not a plus form.

Conjecture 2. For fixed $m$ there are only a finite number of minimal forms.

\section{References}

1. S. Bonan and D. S. Clark, 'Estimates of the Hermite and the Freud polynomials', J. Approx. Theory 63 (1990) 210-224. 307

2. P. Deift, T. Kriecherbauer, K. Mclaughlin, S. Venakides and X. Zhou, 'Strong asymptotics of orthogonal polynomials with respect to exponential weights', Comm. Pure Appl. Math. 52 (1999) 1491-1552. 307

3. P. Deift, T. Kriecherbauer and K. McLaughlin, 'New results for the asymptotics of orthogonal polynomials and related problems via the Lax-Levermore method', Proc. Symp. Appl. Math. 54 (1998). 307

4. P. Deift, T. Kriecherbauer, K. Mclaughlin, S. Venakides and X. Zhou, 'Asymptotics for polynomials orthogonal with respect to varying exponential weights', Internat. Math. Res. Notices (1997) 759-782. 307

5. W. H. Foster and I. Krasikov, 'Bounds for the extreme roots of orthogonal polynomials', Internat. J. Math. Algorithms, to appear. 307, 311

6. I. Krasikov, 'Nonnegative quadratic forms and bounds on orthogonal polynomials', Journal Approx. Theory, to appear. 307, 307, 311

7. I. KRASIKOV and S. LITSYN, 'On the distance distribution of duals of BCH codes', IEEE Trans. Inform. Theory 45 (1999) 247-250. 307

8. A. L. LEVIN and D. S. LuBINSKY, 'Bounds for orthogonal polynomials for exponential weights on $(-1,1)$ and $(-\infty, \infty)$ ', Rend. Circ. Mat. Palermo (2) Suppl. 33 (1993) 75-96. 307

9. A. L. LeVIN and D. S. LubinsKy, 'Asymptotics associated with exponential weights', Internat. Math. Res. Notices 12 (1999) 673-683. 307

10. A. L. LEVIN and D. S. LUBINSKY, 'Bounds for orthogonal polynomials for exponential weights', J. Comput. Appl. Math. 99 (1998) 475-490. 307

11. G. Szegö, Orthogonal polynomials, Amer. Math. Soc. Colloq. Publ. 23 (AMS, Providence, RI, 1975). 307 
Explicit bounds for Hermite polynomials in the oscillatory region

William H. Foster william.foster@brunel.ac.uk

Ilia Krasikov mastiikebrunel.ac.uk

Department of Mathematical Sciences

Brunel University

Kingston Lane

Uxbridge UB8 3PH

London 